\title{
Allgemeine Innere Medizin: Nachwuchs ins Zentrum gerückt
}

\section{Jean-Michel Gaspoz ${ }^{a}$, François Héritier ${ }^{b}$, Drahomir Aujesky $^{c}$, Regula Capauld, Romeo Providolie, Donato Tronnolonef, Franziska Zogg ${ }^{\text {, }}$ Bernadette Häfliger Berger ${ }^{h}$}

a Prof. Dr. med, Co-Präsident SGAIM; 'b Dr. med, Co-Präsident SGAIM; ' Prof. Dr. med, Vorstandsmitglied SGAIM, Leiter der Arbeitsgruppe Nachwuchsförde-

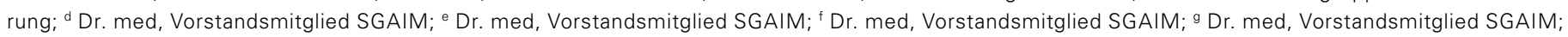
${ }^{\text {h }}$ Rechtsanwältin, MAS NPPM, Generalsekretärin SGAIM

Die Allgemeine Inneren Medizin (AIM) kennt sowohl im ambulanten als auch im stationären Bereich ein Nachwuchsproblem. Aus diesem Grund hat die Schweizerische Gesellschaft für Allgemeine Innere Medizin (SGAIM) ein umfassendes Nachwuchsförderungsprogramm beschlossen. Mit einer Imagekampagne soll der medizinische Nachwuchs gezielt auf die Attraktivität des Generalism aufmerksam gemacht werden.

Gemäss der «Work Force Studie 2015» [1] stellen über 60 Prozent der heute tätigen Hausärztinnen und Hausärzte in den nächsten zehn Jahren ihre Praxistätigkeit altershalber ein. Basierend auf Schätzungen des Schweizerischen Gesundheitsobservatoriums ist damit zu rechnen, dass ohne entsprechende Massnahmen im Jahr 2030 bis zu 40 Prozent der Konsultationen in der ambulanten Grundversorgung nicht mehr abgedeckt werden könnten [2]. Der Öffentlichkeit weniger bekannt ist, dass auch im stationären Bereich in den nächsten Jahren viele erfahrene Chef- und Kaderärztinnen und -ärzte in Pension gehen werden und deshalb ein Mangel an akademisch tätigen Allgemeininternisten/-innen besteht. Ehemals begehrte Chefarztpositionen können oft nur noch mit Mühe besetzt werden.

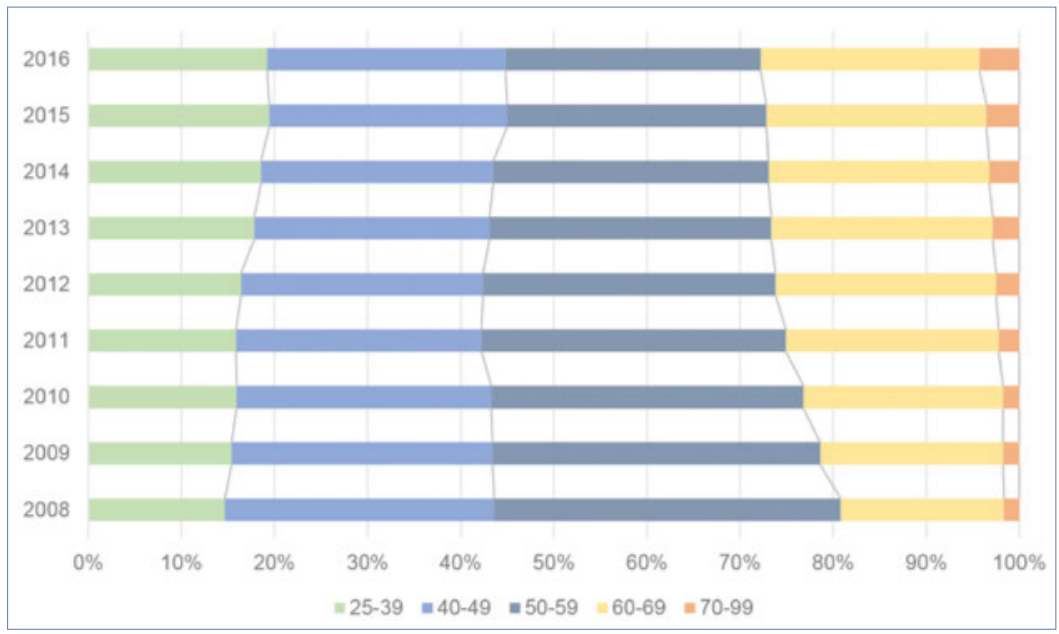

Abbildung 1: Fast 60 Prozent der Allgemeininternisten/-innen sind über 50 Jahre alt.
Eine 2015 vom Berner Institut für Hausarztmedizin durchgeführte Erhebung bei den Jungen Hausärztinnen und -ärzten Schweiz (JHaS) zeigt, dass für die neue Generation - und zwar für Männer wie für Frauen mehr noch als Salär-Bedingungen eine ausgewogene Work-Life Balance zentral ist. Sie wünschen sich mehrheitlich eine Teilzeitanstellung [3]. Diese Ergebnisse werden durch eine repräsentative Onlineumfrage des VSAO Bern vom April 2016 in der Tendenz bestätigt [4]. Knapp ein Drittel der auf diese Umfrage antwortenden Personen, die sowohl im stationären wie auch im ambulanten Bereich tätig sind, wünschen sich eine Teilzeitstelle, wobei als Hauptgrund die bessere Vereinbarkeit zwischen Familie und Beruf angegeben wird. Diese demographischen und gesellschaftlichen Entwicklungen sind eine neue Herausforderung sowohl für die stationäre Versorgung im Spital als auch für die ambulante in der Praxis.

Die immer älter werdende Bevölkerung stellt Gesellschaft und Sozialversicherungen vor neue Probleme. Eine Schätzung der Gesundheitsligen aus dem Jahr 2008 ergab die Zahl von 2,8 Millionen chronischen Krankheitsfällen in der Schweiz [5]. Multimorbidität ist die Regel. Die Begleitung und Behandlung dieser Patienten/-innen verlangt nicht nur einen ganzheitlichen und kontinuierlichen Betreuungsansatz, sondern auch eine enge interdisziplinäre und interprofessionelle Zusammenarbeit. Den Generalisten/-innen wird deshalb zukünftig eine noch zentralere Rolle zukommen. Das Schweizerische Gesundheitswesen ist heute durch seine zunehmende Ausrichtung auf Spezialisierung und der daraus resultierenden Fragmentierung 


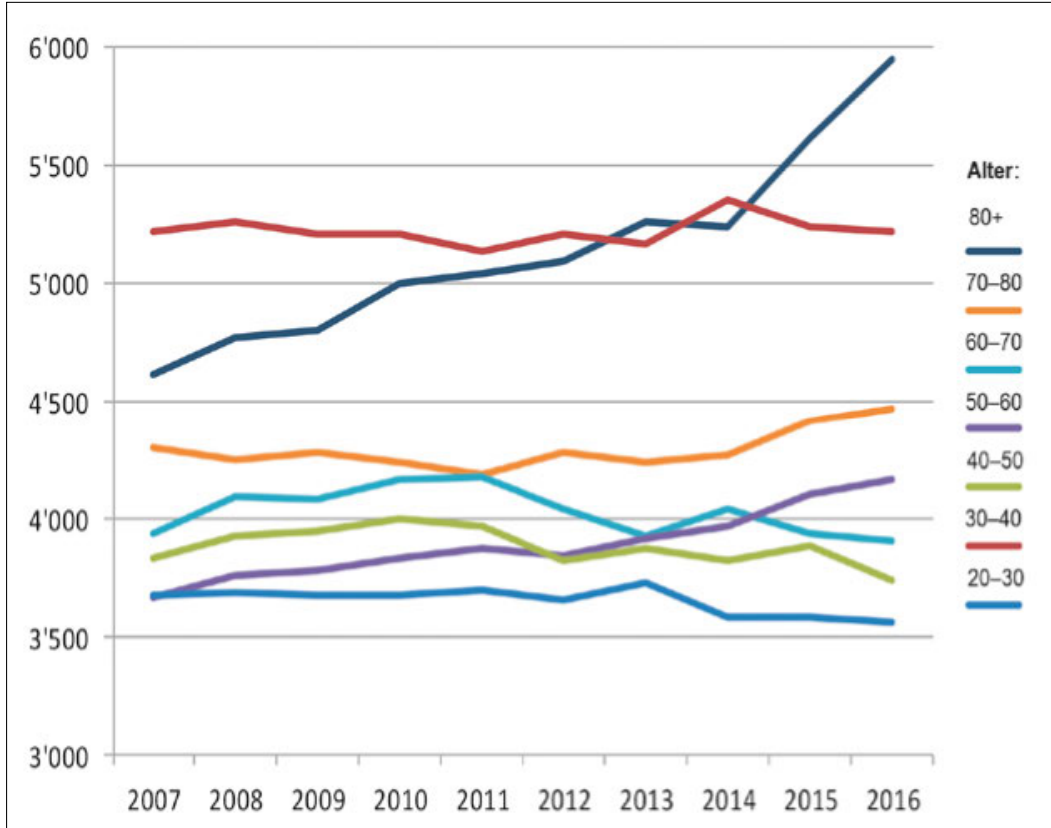

Abbildung 2: Entwicklung des Durchschnittsalters der Patienten/-innen

der Patientenbehandlung, der Weiterbildung und der Lehre suboptimal auf diese Entwicklung vorbereitet. Das Zusammentreffen der drei Faktoren - Demografie, Fragmentierung der medizinischen Versorgung sowie der verstärkte Wunsch nach neuen Arbeitsmodellen bedingt, dass in den nächsten Jahren deutlich mehr Allgemeininternisten/-innen sowohl für den stationären als auch für den ambulanten Bereich ausgebildet werden müssen, damit eine qualitativ hochstehende Basisversorgung in der Schweiz weiterhin flächendeckend garantiert werden kann.

\section{Den Beruf der Allgemeininternisten/-innen attraktiver gestalten}

Die SGAIM hat die Dringlichkeit der Lage erkannt und anlässlich der Delegiertenversammlung 2016 ein grosses Nachwuchsförderungsprojekt lanciert. In den letzten Monaten hat eine eigens dafür eingesetzte Arbeitsgruppe unter der Leitung von Prof. Drahomir Aujesky und Dr. François Héritier zusammen mit Vertretern und Vertreterinnen aus der ambulanten und stationären AIM, den allgemeininternistischen Nachwuchsorganisationen sowie der Akademie eine umfassende Situationsanalyse durchgeführt und verschiedene Empfehlungen formuliert.

Die Literaturauswertung von hauptsächlich aus dem angelsächsischen Raum stammenden Studien zeigt, dass viele intrinsisch-persönliche Faktoren wie tiefere Einkommensvorstellungen, schlechtere Prüfungsno- ten, der Wunsch nach kürzeren Weiterbildungszeiten, fehlendes Forschungsinteresse sowie eine stärkere extraprofessionelle Orientierung mit dem Berufswunsch Generalist/-in verbunden sind. Dies dürfte zumindest teilweise mit dem als tiefer wahrgenommenen Prestige der AIM zu erklären sein. Als Motivationsfaktoren zur AIM hin werden die hohen intellektuellen Herausforderungen, positive Rollenmodelle und Lern- und Weiterbildungserfahrungen sowie ein gutes Mentoring und die berufliche Vielfalt, aber auch die hohe Autonomie genannt. Umgekehrt gibt es Faktoren wie schlechte Praktikumserfahrung und Arbeitsatmosphäre, die z.T. als unattraktiv empfundene komplexe Behandlung von multimorbiden, älteren Patienten/innen, wenig "Action" sowie viel Administration, welche sich auf die Berufswahl negativ auswirken.

Die Arbeitsgruppe zeigt sich davon überzeugt, dass zur Nachwuchsförderung dringend die beruflichen Rahmenbedingungen der AIM attraktiver gestaltet und langfristige Zukunftsperspektiven geschaffen werden müssen. Dazu gehören auch flexiblere Arbeitszeitmodelle wie Jobsharing und Teilzeitstellen. Durch die zunehmende Spezialisierung fand in den letzten Jahren zudem ein schleichender Kompetenzverlust an praktischen Fertigkeiten und die Abwanderung der Forschungsaktivitäten in andere Fachrichtungen statt, was gemäss Empfehlung der Arbeitsgruppe korrigiert werden muss. Die geringere Anzahl akademischer Generalisten/-innen führt bereits in der studentischen Lehre dazu, dass eine Verschiebung vom ganzheitlichen Ansatz zu relativ eng umschriebenen Themen der spezialisierten Medizin stattfindet, was sich unter anderem bei der Berufswahl für den Generalism negativ auswirkt.

\section{Eine den aktuellen Realitäten angepasste Weiterbildung}

In einem komplexen Umfeld ist die Allgemeininternistin, der Allgemeininternist nicht nur für die medizinische Basisbetreuung, sondern auch für die Koordination der interdisziplinären und interprofessionellen Zusammenarbeit zuständig. Grundbedingung hierfür sind breite Kenntnisse, praktische Fertigkeiten und "people skills». Die Arbeitsgruppe empfiehlt eine Überarbeitung des fachlichen Lernzielkatalogs, den Aufbau von systematischen, koordinierten Weiterbildungscurricula und ein aktives Mentoring, damit standardisierte, zur erfolgreichen Berufsausübung relevante Lerninhalte in einer 5-jährigen Weiterbildung auch tatsächlich erlernt und von Generalisten/-innen selbst vermittelt werden können. Gerade im Spital müssen attraktive Berufsprofile für die AIM entwickelt wer- 
den. Da Assistenzärztinnen und -ärzte nur noch etwa einen Viertel ihrer Arbeitszeit am Patientenbett verbringen [6], müssen auch Massnahmen zur Reduktion der steigenden Administrativlast ergriffen werden (z.B. Einstellung von Care-Koordinatoren/-innen, Einführung von nutzerfreundlichen elektronischen Krankengeschichten).

Weil auch Forschung und Entwicklung zentrale Elemente für Perspektiven und Prestige eines Fachgebiets sind, muss in der AIM als zukunftsgerichtetes Fach die Forschung wieder einen höheren Stellenwert erlangen. Dies ist an den universitären Spitälern und Hausarztinstituten durch die Schaffung von akademischen Karrieretracks zu fördern. Hierzu müssen vermehrt Forschungsmöglichkeiten in der AIM selber geschaffen werden, insbesondere in der patientenzentrierten klinischen Forschung. Auch die bisher relativ liberale Praxis der Anerkennung von Weiterbildungsstätten, bei denen allgemeininternistische Weiterbildungsinhalte nicht im Vordergrund stehen, wird überdacht werden müssen.

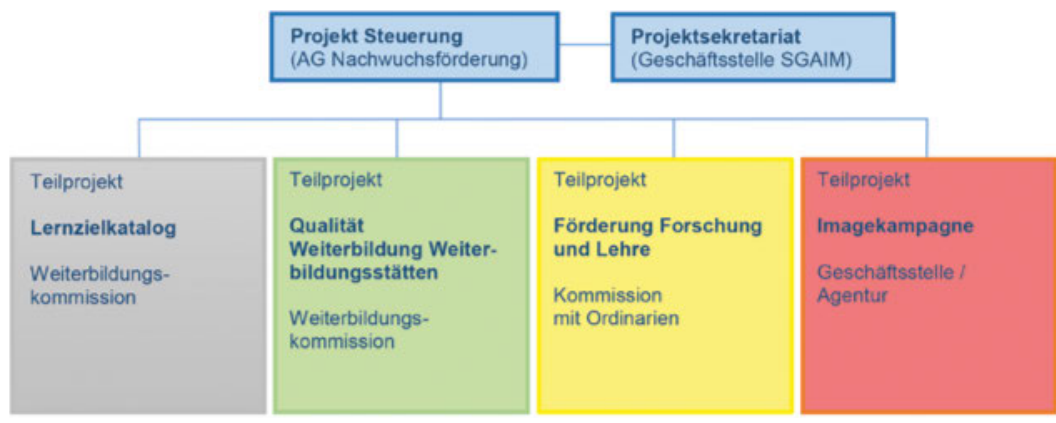

Abbildung 3: Struktur des Nachwuchsförderungsprojekts der SGAIM

\section{Wie weiter: ein Massnahmenkatalog zur Umsetzung}

Die SGAIM wird die Empfehlungen der Arbeitsgruppe in vier Teilprojekten weiterbearbeiten und konkrete Massnahmen vorschlagen bzw. umsetzen:

Weitere Schwerpunkte sind die Unterstützung und die Förderung der Nachwuchsorganisationen der SGAIM, der JHas und der im letzten Jahr neu lancierten Swiss Young Internists (SYI), welche mit ihrer Vereinsaktivität die Attraktivität der stationären Inneren Medizin bekannter machen und ein Netzwerk für junge Assistenzärzten/-innen AIM aufbauen will. Der nächste Frühjahrskongress der SGAIM (30. Mai bis 1. Juni 2018 in Basel) ist ebenfalls dem Thema "Nachwuchs - unser Kapital» gewidmet und richtet sich in speziellen Themennachmittagen auch an das studentische Publikum und die Assistenzärzte/-innen. Neu werden am Frühjahrskongress auch akademisch Interessierte an sogenannten "Meet-the-Professor»-Treffen und durch methodologische Seminare (grant writing course) gefördert. Die SGAIM Foundation unterstützt zudem mit ihrer diesjährigen Preisausschreibung die Forschung zum medizinischen Nachwuchs.

\section{Werbekampagne}

Die Faszination des Berufs der medizinischen Allrounder/-innen - sei es im ambulanten oder im stationären Bereich, aber auch in der Wissenschaft und der Forschung - soll im Zentrum einer Werbekampagne der SGAIM stehen. Dabei ist der Auftritt der Kampagne unter dem Slogan "Ärztin/Arzt. Alle anderen sind Spezialisten.» bewusst etwas provokativ gewählt. Die in dieser Woche lancierte Kampagne wird mit Aktivitäten online auf Social-Media-Kanälen, mit Printsets für Ärztinnen und Ärzte sowie mit einer vertieften thematischen Auseinandersetzung, zum Beispiel an Kongressen, aufzeigen, wie vielseitig und attraktiv die Aufgaben und Perspektiven der Fachärzte/-innen AIM in der medizinischen Basisversorgung sind, unabhängig davon ob sie im Spital, in der Wissenschaft oder in der Hausarztmedizin geleistet wird.

\section{Bildnachweis}

Abbildung 1: FMH-Ärztestatistik (http://aerztestatistik.myfmh2.fmh. ch, Stand: 13.02.2018)/SGAIM

Abbildung 2: Hopitaux Universitaires Genève Abbildung 3: SGAIM

Referenzen - Endnotes:

1 Zeller A, Tschudi P. "Anamnese und Status» bei Schweizer Hausärzten. Primary and Hospital Care 2016;16(15):277-80.

2 Senn N, Ebert ST, Cohidon C. (2016). La médecine de famille en Suisse. Analyse et perspectives sur la base des indicateurs du programme SPAM (Swiss Primary Care Active Monitoring) (Obsan Dossier 55). Neuchâtel: Observatoire suisse de la santé.

3 Gisler L, Streit S. «Erst angestellt, dann selbständig in ärzteeigenen Gruppenpraxen», Schweizerische Ärztezeitung, 2017;98:09

4 Hess B. Ein Drittel will Teilzeit arbeiten. VSAO JOURNAL 2016, Nr. 2, unter https://vsao-bern.ch/de/downloads/sektionsnach richten/2016/april-teilzeit-arbeiten.pdf [Stand: 13.02.2018]

5 Oggier W. Gesundheitswesen Schweiz 2015-2017, Hogrefe Verlag; 2015., 5. Auflage, S. $82 \mathrm{ff}$.

6 Wenger N et al. FM276: Quantitative and objective assessment of resident's workday organization: the Medical Day study (abstract). Swiss Primary and Hospital Care, 2016. 16 Suppl (9): p. 21-2. 